\title{
Rabies Virus
}

National Cancer Institute

\section{Source}

National Cancer Institute. Rabies Virus. NCI Thesaurus. Code C112405.

A species of enveloped, rod- or bullet-shaped viruses in the Rhabdoviridae family and Lyssavirus genus. The genome is composed of single-stranded, negative-sense RNA. Rabies viruses are neurotropic and zoonotic. They are found in mammals and humans and causes acute infection of the central nervous system, neural degeneration, and fatal encephalitis in humans. Rabies viruses are transmitted via direct contact with saliva from infected animal or human bites. 